Sartre, la médecine et le procès d'infanticide de Liège : De la vie à l'Histoire

\title{
Grégory Cormann
}

Université de Liège

gregory.cormann@uliege.be

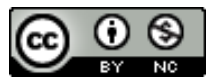

\section{Résumé}

Le rapport de Sartre à la médecine a échappé à la critique. Son désintérêt pour les sciences justifierait l'absence dans son œuvre d'une réflexion détaillée sur la médecine ou sur la maladie. La publication d'inédits sur la morale écrits entre 1961 et 1965 , lorsque s'achève la Guerre d'Algérie, demande de considérer cette question à nouveaux frais. Dans ces inédits, notamment dans Les racines de l'éthique, la question des attitudes devant la vie et devant la mort reçoit une attention décisive. On s'intéressera ici à la réaction de Sartre au procès célèbre d'infanticide qui s'est tenu à Liège en 1962, aussi connu comme le Procès du Softénon. Prenant position contre les commentaires des revues de gauche de l'époque, Sartre y déploie une attention scrupuleuse aux transformations biopolitiques que connaissent alors les sociétés occidentales. Cette attention s'inscrit, sur le fond de son histoire familiale, dans un dialogue passé inaperçu avec l'École des Annales, avec l'histoire des attitudes devant la vie et devant la mort de Philippe Ariès, mais aussi avec l'histoire des sensibilités de Lucien Febvre.

Mots-clé: Sartre, Procès du Softénon, École des Annales, Ariès, Guerre d'Algérie

\begin{abstract}
Sartre, Medicine, and the Infanticide Trial in Liège: From Life towards History

Sartre's attitude toward medicine has been neglected by researchers, insofar as his disinterest in sciences would justify the absence in his work of a thorough reflection on medicine or disease. The publication of some unpublished works on morals written
\end{abstract}

ISSN: 0874-9493 (print) / ISSN-e: 2183-0142 (online)

DOI: 10.2478 /phainomenon-2018-0016

(C) 2018 Cormann. This is an open access article licensed under the Creative Commons Attribution-NonCommercial-NoDerivs License (http://creativecommons.org/licenses/by-nc$\mathrm{nd} / 3.0 /)$. 
between 1961 and 1965, when the war of Algeria was coming to an end, asks to reassess this issue. In these unpublished works, especially in Les racines de l'éthique, the issue of attitudes toward life and death draws significant attention. In this article, we dwell upon Sartre's reaction to the famous infanticide trial held in Liège in 1962, also known as the Softenon Trial. Taking a stand against the accusatory comments of leftist journals, Sartre pays scrupulous attention to the biopolitical transformations that Western societies are then experiencing. This attention should be understood in the background of Sartre's family history as well as in an unnoticed dialogue with the School of Annales, with the history of attitudes towards life and death delineated by Philippe Ariès and also with the history of sensibilities outlined by Lucien Febvre.

Keywords: Sartre, Softénon Trial, School of Annales, Ariès, War of Algeria

Le rapport de Sartre à la médecine a été très peu étudié. Cette indifférence au rapport de Sartre à la médecine s'explique certainement par la représentation que l'on se fait de la phénoménologie existentialiste sartrienne, apparemment plus soucieuse de la philosophie et de la littérature que des sciences et des savoirs médicaux. Le contraste entre littérature et médecine semble trouver une illustration exemplaire dans L'Idiot de la famille, la biographie que Sartre consacre à Flaubert au début des années soixante-dix (Sartre 1971-72) : Gustave Flaubert, avant de devenir un des grands représentants de la littérature française, fut d'abord le raté de la famille des médecins Flaubert, père et fils, le pater familias Achille-Cléophas et son fils aîné Achille. Une telle opposition ne peut cependant pas être considérée de façon unilatérale : on ne peut en effet que s'étonner de l'intérêt que Sartre a toujours porté pour des auteurs ou des écrivains qui avaient une formation de médecins. On ne saurait surestimer l'importance pour Sartre de Freud, de Ferenczi ou de Lacan, de Jaspers, de Minkowski ou de Lagache, de Georges Duhamel, de Céline ou de Jean Delay, de Frantz Fanon ou de Carlo Levi. Sans oublier la figure tutélaire d'Albert Schweitzer, son cousin maternel, le célèbre Docteur Schweitzer, qui reprit des études de médecine après s'être d'abord illustré en philosophie, en théologie et en musicologie. À considérer cette suite de noms, on pourrait dire que, pour Sartre, il n'y a rien de tel que la médecine à condition d'en sortir. Relayée par la psychanalyse, la littérature ou l'engagement politique, la médecine, telle qu'elle semble avoir accompagné l'ensemble de l'entreprise de Sartre, se mue en moyens d'analyse et de contestation social et politique. 
Avant Foucault, Sartre se montre ainsi sensible au développement, tout au long du XIX ${ }^{\text {ème }}$ siècle, de nouvelles technologies de pouvoir biopolitiques libérales. Celles-ci apparaissent comme une grande médecine sociale qui envahit l'ensemble de l'existence et qui a vocation à gérer et à exploiter les populations de la manière la plus rentable. La médecine - et les médecins entre ainsi sur la scène politique. Cette «médecine sociale» prend alors différentes formes, comme la médecine préventive ou les sciences de l'éducation ${ }^{1}$. Dans Questions de méthode, Sartre évoque cette transformation biopolitique de la société, en citant d'autres figures de la pratique médicale, comme les médecins militaires et les médecins coloniaux. Par cette nouvelle forme de gouvernementalité, la médecine fournit ainsi l'exemple de ce que Sartre définit comme une (nouvelle) possibilité sociale, à savoir une forme d'existence qui offre à ceux qui peuvent y accéder un avenir qui, d'un coup, une fois pour toutes, « ouvre [leur vie] jusqu'à la mort ».

Il faut donc concevoir la possibilité comme doublement déterminée : d'une part c'est, au cœur même de l'action singulière, la présence de l'avenir comme ce qui manque et ce qui dévoile la réalité par cette absence même. D'autre part, c'est l'avenir réel et permanent que maintient et transforme sans cesse la collectivité : lorsque les besoins communs entraînent la création de nouveaux offices (par exemple, la multiplication des médecins dans une société qui s'industrialise), ces offices non encore remplis - ou vacants par suite des retraites, des morts - constituent pour certains un avenir réel, concret et possible : ils peuvent faire leur médecine, la carrière n'est pas encombrée ; du coup, leur vie s'ouvre jusqu'à la mort : toutes choses égales d'ailleurs, les professions de médecin militaire, de médecin de campagne, de médecin colonial, etc., sont caractérisées par certains avantages et certaines obligations qu'ils connaîtront vite. Cet avenir, bien sûr, n'est que partiellement vrai : il suppose un statu quo et un minimum d'ordre (exclusion des hasards), ce que contredit justement l'historialisation constante de nos sociétés. Mais il n'est pas faux non plus puisque c'est lui - autrement dit les intérêts de la profession,

\footnotetext{
${ }^{1}$ Sur cette histoire de la médecine, je suis redevable à la thèse d'Elsa Roland (Roland, 2017). Je retiendrai ici, en-deçà des questions pédagogiques, la prise en charge de la vie humaine dès le plus jeune âge par la médecine hygiéniste qui fait des mères - et de la relation entre les mères et leurs nouveau-nés - un levier majeur de l'organisation sociale.
} 
de la classe, etc., la division toujours plus poussée du travail, etc. - qui manifeste d'abord les contradictions présentes de la société. Il se présente donc comme possibilité schématique et toujours ouverte et comme action immédiate sur le présent. (Sartre, 2005a : 88)

Les exemples pris par Sartre : les professions de médecin militaire, de médecin de campagne, de médecin colonial, méritent un peu d'attention. Leurs référents immédiats sont assez évidents. Outre l'allusion transparente à Flaubert, vers lequel Sartre tourne déjà son attention en 1957, on peut penser que, lorsqu'il évoque les figures du médecin colonial et du médecin militaire, il fait allusion, d'une part, à Albert Schweitzer, Prix Nobel de la Paix 1952 pour son œuvre à l'hôpital de Lambaréné autant que pour son combat pour la paix après les deux guerres mondiales, et, d'autre part, allusion à la Guerre d'Algérie dont les violences s'inscrivent, en cette année 1957, dans les corps et dans les esprits des colonisés en lutte comme dans ceux des soldats du contingent envoyés en Algérie. Toutefois, quand on y prend garde, ces exemples semblent aussi avoir une résonance très personnelle. Elle concerne Sartre directement, dans son rapport à sa famille paternelle : son grand-père Eymard Sartre était docteur à Thiviers, en Dordogne ; son père, Jean-Baptiste, mourut en 1906 dans la même ville d'une fièvre attrapée en Cochinchine, là où l'avait attiré son rêve de devenir marin ${ }^{2}$. Aux antipodes l'une de l'autre, on retrouve là, très simplement opposée, la figure du médecin de campagne et les figures du médecin militaire/colonial. Mais on peut penser qu'elle concerne encore davantage Sartre de façon indirecte, par le truchement de Merleau-Ponty, qui est son

\footnotetext{
${ }^{2}$ Annie Cohen-Solal a retracé la trajectoire d'ascension sociale de la famille Sartre au cours du XIX ${ }^{\text {ème }}$ siècle dans leurs terres de Thiviers, dans le Périgord, sur trois générations, d'un petit fermier au médecin de campagne Eymard Sartre : «Un fermier engendra un gros métayer qui engendra un médecin de campagne ». Cette success story exemplaire, tout à la fois accomplie par Jean-Baptiste et recouverte par sa mort prématurée, A. Cohen-Solal veille à la remettre dans le contexte de l'histoire de la France post-révolutionnaire qui voit Eymard Sartre transcender ses origines paysannes par une union (malheureuse, car socialement impossible) avec Élodie Chavoix, descendante de l'élite républicaine fortunée locale, les Theulier et les Chavoix notamment, qui avaient « fourni à la France, depuis la Révolution de 1789, un nombre impressionnant de maires, de sous-préfets, de conseillers généraux, de députés qui représentèrent la Dordogne ou le Périgord auprès des assises nationales. » (CohenSolal, 1999 : 31-36, 34 et 31 en ce qui concerne les deux extraits cités).
} 
interlocuteur principal dans Questions de méthode. Emmanuel Alloa a récemment insisté sur l'histoire coloniale de la famille de Merleau-Ponty ${ }^{3}$. Son grand-père Samuel Anatole Mazeppa Merleau-Ponty fut médecin en NouvelleCalédonie pendant 30 ans. Son oncle Jean Jules Merleau-Ponty fut médecin de marine, notamment affecté à Madagascar, où le propre père de Merleau-Ponty, Bernard, militaire, fit une partie de son service d'officier. Alloa précise que, de retour en Métropole, Bernard Merleau-Ponty, qui meurt en 1913, est un des premiers patients du médecin et psychanalyste Angelo Hesnard « qui crée dès 1912 à l'Hôpital maritime de Rochefort un service de neuropsychiatrie freudienne pour soigner certains marins de retour de Cochinchine ou de Madagascar, souffrant de paludisme et parfois assujettis aussi à l'opium, aux drogues, à l'alcool et aux jeux d'argent » (Alloa, 2018 : 118). L'histoire ne peut manquer d'attirer l'attention: alors qu'en 1956-1957 Merleau-Ponty retourne sur les traces de cette histoire, en faisant de longs séjours en Afrique et à Madagascar, Sartre, qui ne peut ignorer cette histoire, déplacerait ainsi le terrain de leur querelle vers une histoire commune qui les réunit irrémédiablement du point de vue d'une mort paternelle contractée dans les lointaines terres coloniales françaises ${ }^{4}$.

Considéré au prisme de la relation de Sartre avec Merleau-Ponty, la question de la médecine endosse donc une double fonction synthétique. D’un côté, elle articule les niveaux sociaux macro et micro : la transformation biopolitique de la société que Sartre signale dans Questions de méthode se rejoue au niveau de trajectoires sociales singulières qui sont celles des familles Sartre et Merleau-

\footnotetext{
${ }^{3}$ S'agissant de Merleau-Ponty, voir Alloa, $2018: 117-118$. E. Alloa s'appuie, de façon décisive, sur les «Entretiens avec Georges Charbonnier » (1959) de Merleau-Ponty, récemment republiés, qui éclairent l'importance pour le philosophe des conférences qu'on lui propose de faire, en 1956-1957, à travers ce qui reste alors l'espace colonial français. Voir Merleau-Ponty, 2016.

${ }^{4}$ Témoignant des rencontres privées qu'ils ont eues entre 1956 et 1961, Sartre évoque les discussions qu'il eut alors avec Merleau-Ponty concernant la Guerre d'Algérie. Malgré le climat de brouille, peut-être grâce à lui, Sartre insiste sur ce qui les réunissait alors : « Nous étions d'accord pour condamner sans réserve la guerre d'Algérie [...]; peut-être n'étions pas du même avis sur les moyens de lutter contre elle ; cela viendrait : quand il monte, le fascisme rejoint les amis perdus » (Sartre, 2010a : 1116).
} 
Ponty ${ }^{5}$. Mais, d'un autre côté, on voit aussi que l'exemple de la médecine appliqué à ces deux espaces familiaux permet tout à la fois de considérer ses effets du point de vue subjectif et du point de vue objectif. Les Sartre comme les Merleau-Ponty sont en effet à la fois sujets de la médecine, dans la mesure où ils donnent à la profession un certain nombre de ses membres, et objets de la médecine (et de la maladie), en tant qu'ils sont, sinon les victimes, du moins les objets de pratiques médicales (inefficaces) ${ }^{6}$.

Dans le cadre de cet article, je vais essayer de préciser cette relation de distance et de proximité de Sartre à l'égard de la médecine à partir de l'étude d'un cas, un cas d'infanticide qui a été au cœur de l'actualité judiciaire, sociale et intellectuelle (à gauche) au début des années 1960 : le Procès de Liège, qui a été analysé par Sartre de façon répétée dans plusieurs textes posthumes des années 1960 que j'ai eu l'occasion de publier ces dernières années.

Le Procès de Liège, aussi connu sous le nom de Procès du Softénon, est un procès d'assises qui a eu lieu à Liège, en Belgique, du 5 au 11 novembre 1962. Un jury populaire avait à juger de la culpabilité de plusieurs membres de la famille Vandeput et de leur médecin de famille, le docteur Casters, accusés d'avoir mis fin aux jours, par empoisonnement, de la fille du couple Vandeput, âgée de 8 jours, qui était née avec de lourdes déformations physiques. Ayant essuyé le refus des médecins hospitaliers d'euthanasier leur fille, les parents de la petite Corinne avaient administré à leur fille un cocktail létal fourni par le médecin de famille. Les parents estimaient que leur fille ne pourrait pas avoir une vie humaine vivable et avait donc décidé de lui donner la mort eux-mêmes ${ }^{7}$.

L'affaire a suscité un grand émoi dans la société belge, et trouvé de nombreux échos dans l'opinion publique des pays occidentaux (en France, en Allemagne et aux États-Unis notamment) : les déformations physiologiques de

\footnotetext{
${ }^{5}$ Sartre fait ainsi droit ici à la médiation de la famille, comme de façon générale dans Questions de méthode, à la suite de l'école durkheimienne.

${ }^{6}$ Pour Sartre, la question du rapport à la vie se fait d'emblée sur un mode problématique et médicalisé : dans sa prime enfance, la santé de Sartre inquiète sa famille à Thiviers, alors que, dans le même temps, son père combat la fièvre qui va bientôt l'emporter.

7 À l'époque, plusieurs ouvrages journalistiques ont été consacrés à l'affaire. Voir Paulus et Rozet, 1963 ; Toussaint, 1963. Le procès de Liège a aussi fait l'objet, il y a une vingtaine d'années, de deux mémoires de fin d'études de Valérie Warowny à l’Université de Liège (Warowny, 1999 ; Warowny, 2002).
} 
l'enfant étaient dues à l'ingestion par la mère d'un médicament, le Softénon, qui était alors prescrit aux femmes enceintes afin d'apaiser les nausées et les stress du début de grossesse. L'année précédente, un médecin australien avait fait la relation entre ce médicament - et sa molécule active, la thalidomide -, et les malformations monstrueuses des membres et/ou des viscères qui affectaient les enfants des mères qui avaient utilisé ce produit mis en circulation quelques années plus tôt. Suscitant la polémique, certains ont parlé de ces enfants Softénon comme des « bébés-phoques » à cause des moignons qui leur tiennent lieu de membres supérieurs et qui les rendent reconnaissables.

Contrairement à d'autres interventions intellectuelles concernant l'affaire, il n'est jamais question pour Sartre de condamner, de quelque manière que ce soit, le comportement de la famille. Je fais l'hypothèse que Sartre analyse l'infanticide de Liège afin de mettre en évidence l' « historialisation constante » des sociétés, dont il parle dans Questions de méthode, précisément à partir d'un exemple qui relève de la médecine, c'est-à-dire à partir de la pratique humaine qui concerne le plus directement notre rapport à la vie et à la mort. Si cela est vrai, Sartre aurait ainsi trouvé les moyens de confronter son lecteur (finalement posthume) à la présentation d'une possibilité pure au lieu de se contenter de dénoncer une nouvelle forme de normativité sociale. Plutôt que d'aborder des travaux théoriques ou des études de cas qui relèveraient de façon canonique de la psychologie ou de la psychopathologie phénoménologique, l'enjeu de cet article sera donc de faire faire l'épreuve d'un possible à l'état pur là où l'expérience sociale ne verrait (et n'a vu) que folie individuelle ou pathologie sociale.

\section{Les Temps Modernes et Esprit face aux accusés du procès de Liège : du soutien populaire à la mise en cause par les intellectuels de gauche}

Le procès de Liège fait grand bruit ${ }^{8}$. L'opinion publique suit l'affaire de près. Une grande foule est massée autour du Palais de justice de Liège pour attendre

\footnotetext{
${ }^{8}$ Le 17 novembre 1962, quelques jours après la fin du procès, Paris-Match consacre sa Une à l'affaire avec une photo de la maman couvrant l'entièreté de la couverture du magazine. La légende se veut interrogative : «En votre âme et conscience, comment l'auriez-vous jugée? » L'effet majeur de cette couverture, à travers cette question
} 
le prononcé du verdict. La foule réagit certes avec enthousiasme au verdict d'acquittement de la Cour d'assises. Mais cet enthousiasme ne dure pas. Suite à l'acquittement, la réaction populaire est marquée par une contradiction. Bien sûr, avant le procès, l'affaire avait vu s'opposer la gauche (progressiste) et la droite (catholique) qui voyait dans le geste des parents une atteinte intolérable au respect sacré de la vie. Mais, à l'issue du procès, le retournement de l'opinion suscite d'autres questions : ceux-là mêmes qui avaient soutenu la famille avant le procès, une bonne partie de l'opinion publique, la presse de gauche et des intellectuels de gauche, se montrent plus critiques et regrettent que l'infanticide n'ait pas fait l'objet d'une condamnation, même symbolique. Ainsi, ceux-là mêmes qui étaient favorables aux accusés avant et pendant le procès, quand ils pensaient que ceux-ci allaient être condamnés, regrettent, une fois que l'acquittement a été prononcé, que les mêmes personnes n'aient pas eu à endosser une partie de la culpabilité de la mort de la petite Carine. Tout se passe comme si l'attitude des parents apparaissait au public et à la presse (intellectuelle) de gauche comme relative (et réductible), elle aussi, aux transformations récentes des sociétés occidentales marquées par les valeurs bourgeoises de rentabilité (limitation des naissances et recherche de la réussite sociale pour ses enfants sur fond d'eugénisme social) et par un développement technique et technologique qui envahit tous les aspects de l'existence, y compris ce qui relève du corps humain, de sa vie et de sa mort (Domenach et Marie, 1962).

Deux raisons me poussent à revenir aujourd'hui sur ce procès qui a été célèbre. Deux raisons qui font que cette affaire, qui a marqué les esprits sur le moment, a eu des suites. La première raison est que cette histoire continue : les victimes du Softénon ont aujourd'hui une soixantaine d'années. Depuis quelques années, leur situation fait régulièrement retour dans l'actualité médiatique et politique en Belgique. Les victimes du Softénon qui ont survécu (la fille du couple Vandeput aurait eu une chance sur dix de survivre à ses pathologies) sont aujourd'hui confrontées à un moment critique de leur vie : leur âge suppose de nouvelles prises en charge sociales alors même que leurs parents sont aujourd'hui très âgés ou décédés. L'aide familiale dont ils ont

apparemment anodine, est la restriction de l'enjeu social et politique au jugement de la mère. 
bénéficié depuis toutes ces années n'est donc plus assurée à une période critique de leurs vies. La question d'une responsabilité sociale (publique) est donc de nouveau posée (à défaut d'interventions financières des sociétés héritières de l'entreprise pharmaceutique qui a commercialisé le Softénon dans les années 1950). Depuis 2011, plusieurs demandes ont été adressées aux autorités politiques belges, suivies d'un engagement du gouvernement fédéral de créer un Fonds d'utilité publique doté de 5 millions d'euros afin d'indemniser les personnes lourdement handicapées. Suite à un changement de majorité, le fonds n'a jamais été créé. Très récemment, en février 2018, l'État belge a été condamné par le Conseil d'État à constituer ce fonds. Aujourd'hui encore, par conséquent, des conditions de vie décentes ne sont toujours pas assurées pour les victimes du Softénon et leur statut de victimes d'un scandale médical (le risque de déformations était connu de la firme pharmaceutique) n'est toujours pas effectivement reconnu (Dupont, 2018).

La deuxième raison qui m'amène à reprendre le dossier est le fait que l'infanticide de Liège a été étudié par Sartre dans une série de textes sur la morale, restés complètement inédits jusqu'il y a quelques années (et qui restent partiellement inédits), que le philosophe écrit de façon soutenue entre 1963 et 1965 pour deux conférences (une conférence qu'il fait à Rome, à l'Institut Gramsci, en mai 1964 ; une conférence qu'il devait faire au printemps 1965 à Cornell, qu'il annulera en signe de protestation contre la guerre du Vietnam) ${ }^{9}$. Ce qui m'intéresse ici, c'est le rapport particulier de Sartre à ce procès. Sartre ne s'est pas directement exprimé sur l'affaire. En revanche, Les Temps Modernes l'ont fait, une fois, après le procès, en février 1963, au travers d'un entretien entre J.-B. Pontalis, qui s'occupait alors de la revue, et Françoise Dolto (Pontalis et Dolto, 1963). Les deux psychanalystes, peut-être influencés par la Une de Paris-Match, se montrent extrêmement sévères à l'égard du comportement «agressif» de la mère à l'encontre de sa fille. Le point de vue « officiel » des Temps Modernes pose question : d'abord, parce qu'alors qu'elle est en retard sur l'événement, la position que prend la revue est en porte-à-faux avec son inspiration phénoménologico-existentialiste ; elle se limite de fait à

${ }^{9}$ Les racines de l'éthique (Sartre, 2015); «Morale et Histoire» (Sartre, 2005b). S'agissant de cette dernière conférence, la plupart des développements concernant le Softénon sont encore inédits. 
des réflexions générales d'inspiration psychanalytique sur les relations familiales contemporaines, à partir de comparaisons psychopathologiques (les patients et patientes de F. Dolto). Ensuite, parce que la position des Temps Modernes se fait l'écho et amplifie la contradiction idéologique dans laquelle la pensée de gauche est plongée pendant l'hiver 1962-1963. Dans Les Temps Modernes, comme dans Esprit, le moment est à la condamnation des accusés et, en particulier, de la mère accusée. C'est de cette contradiction que Sartre part dans Les racines de l'éthique: «Journaux français de gauche: pour pendant le procès, contre au moment de l'acquittement » (Sartre, $2015: 26$ ).

Lorsque Sartre évoque le changement d'avis de la presse et des intellectuels de gauche, sa cible implicite évidente est le numéro de la revue Esprit qui a traité de la question en décembre 1962. Cependant, comme nous allons le voir, si Sartre vise peut-être les hésitations de la revue créée par Mounier, cette critique se fait sur la base d'un accord fondamental concernant l'importance d'une étude de la société qui accorde une place importante au rapport médical et à ses techniques. En revanche, le travail que Sartre fait sur l'exemple du Softénon est une critique implicite mais frontale des propos de Pontalis et de Dolto dans Les Temps Modernes.

En ce qui concerne Esprit, la revue prend position à la suite d'un premier texte (court) de Pierre Marie, antérieur au Procès de Liège. L'article, intitulé «Les bébés phoques » (Marie, 1962), fait scandale auprès des lecteurs tant par son contenu, qui envisage la possibilité de l'avortement ou de l'euthanasie dans cette situation, que par son titre qui apparaît en lui-même dégradant et inapproprié dans les pages de la revue du grand Emmanuel Mounier. Deux mois plus tard, après les explications de Pierre Marie, Jean-Marie Domenach, le directeur de la publication, s'exprime au nom de la revue. Il défend certes l'article initial de Pierre Marie et rappelle qu'il ne s'agit pas de s'offusquer à propos d'un cas, mais bien de prendre en charge les 10000 cas d'enfants victimes de la thalidomide, que l'on serait bien incohérent de laisser vivre si on ne se donne pas les moyens de les prendre en charge. Mais, en réalité, Domenach glisse subrepticement de cette vigilance sociale à une critique généralisée de la société. Cela lui fait considérer l'infanticide de Liège comme le symbole d'une société tournée vers le bonheur (individuel, superficiel) qui « fait tout pour expulser de son sein, pour radier définitivement de la famille le 
petit être qu'elle a laissé déformer » (Domenach et Marie, $1962: 1054)^{10}$. En conclusion, le directeur d'Esprit fustige « l'hystérie des réactions populaires au procès de Liège » et en appelle à une autre morale qui soit capable de « défendre la vie par d'autres moyens que le meurtre, ce retour aux pratiques primitives de la vie naturelle » (Domenach et Marie, 1062 : 1055). Le compte est bon.

S'agissant des Temps Modernes, la charge est encore plus virulente. Interrogée par Pontalis, F. Dolto passe sans transition d'une première réaction qui l'avait amenée à admirer « l'amour fantastique » dont Mme Vandeput avait dû faire preuve, à une critique des « désirs agressifs, destructeurs, pathogènes » que les mères sont capables de manifester (Pontalis et Dolto, 1963 : 12721273). La psychanalyse est servie à la hache. «Les mères » sont clouées au pilori, tantôt capables de faire de leur enfant un objet de maîtrise et de jouissance, tantôt de l'abandonner à qui voudrait s'en occuper, tantôt capables d'éloigner les pères de leur responsabilité parentale, tantôt de se faire faire un enfant pour assouvir les désirs qu'elles n'ont pas pu assouvir elles-mêmes. Pontalis et Dolto font ainsi le portrait délirant d'une société moderne qui a produit à vaste échelle une « maternité pathologique » (Pontalis et Dolto, 1963 : 1280).

Dans ce contexte, la position de Sartre (restée inédite, mais on sait que Sartre a longtemps pensé publier en volume le texte écrit pour sa conférence annulée à Cornell) se distingue carrément de ce qu'on peut alors lire dans les milieux intellectuels de gauche. D'une part, Sartre cherche à comprendre ce retournement subit de l'opinion. Comment se fait-il que la demande (compréhensive) d'acquittement se soit transformée aussi brusquement en une condamnation morale (sévère) ? D'autre part, Sartre s'inscrit en faux contre la réduction de la décision de donner la mort à la petite fille à une explication sociologique. S'il ne peut se comprendre, comme nous le verrons, que dans un monde qui accorde une place de plus en plus grande à la technique et à l'action

\footnotetext{
${ }^{10}$ La société refuse ainsi de voir - et se donne le moyen de ne pas voir - ce qui est son produit. J.-M. Domenach rapproche alors explicitement la complaisance pour l'infanticide de Liège de l'indifférence des hommes et des femmes qui ont « détourn[é] les yeux en passant devant le corps de l'Algérien que la police laissait agoniser sur le trottoir parisien, le 17 octobre de l'année passée. » (Ibid.) Domenach fait allusion à la répression policière de la manifestation des Algériens à Paris le 17 octobre 1961.
} 
sur la vie humaine, l'acte des parents liégeois est selon Sartre un acte respectueux de la vie et, pourrait-on dire, de l'humanité de la vie humaine :

fondamentalement, l'infanticide de Liège est un acte qui dénonce à travers un cas particulier notre société comme invivable, au nom de notre avenir inconditionné. Cet acte n'est pas une simple dénonciation : puisque l'avenir inconditionné ne peut être objet de connaissance, c'est en se constituant comme moyen de rapprocher l'avenir qu'il dénonce le présent par le surgissement même de l'avenir rapproché. (Sartre, 2015 : 42)

Sartre nous oblige ainsi à nous poser une seconde question. Comment peut-on penser un respect de la vie dans la mort donnée ? Il y a pour Sartre des vies qui ne sont rien d'autre qu'une mort interminable, une mort-dans-la-vie ${ }^{11}$. Dans la situation-limite qui constitue le fond du procès de Liège, il faut aussi pouvoir penser une vie-dans-la-mort.

\section{Sartre et la revue Esprit : la politique par d'autres moyens}

Dans Les racines de l'éthique, le texte qu'il a écrit pour sa seconde conférence à l'Institut Gramsci, en 1964, Sartre utilise deux exemples principaux : l'exemple du racisme colonial, d'une part, celui du procès de Liège, d'autre part. Les deux exemples semblent dans un premier temps n'avoir rien de commun. Qu'est-ce qui peut bien rapprocher l'expérience de la domination coloniale, que Sartre décrit à partir des Damnés de la terre de Frantz Fanon, et une affaire judiciaire en Belgique ? En réalité, il semble bien que Sartre ait choisi deux exemples - l'un macro, qui relève de la géopolitique, l'autre micro, qui relève de la décision individuelle et quasiment de l'intime -, mais qui, dans les deux cas, exposent l'être humain à une impuissance radicale et à la violence : d'un côté, le racisme qui fait de certains hommes des sous-hommes plongés dans un monde de violences qu'ils subissent en les retournant le plus souvent contre eux-mêmes; de l'autre, les bébés Softénon que la prise en

${ }^{11}$ Le thème a été développé avec beaucoup de force par Fanon dans Les Damnés de la terre (Fanon, 2011) et dans ses travaux psychiatriques (Fanon, 2015). 
charge médicalisée des naissances confronte à la mort ou à la perspective d'une vie invivable ${ }^{12}$.

Le rapprochement ici esquissé ne doit cependant pas être précipité. En effet, si dans sa conférence de 1964 Sartre revient sur la «révolution algérienne » (Sartre, 2015 : 54), c'est pour soumettre sinon son enthousiasme, du moins son engagement de 10 années pour l'indépendance de l'Algérie, au bilan négatif des premières années d'indépendance. L'exploitation des anciennes colonies continue. Ce n'est pas à un tel bilan négatif que Sartre veut faire servir l'exemple du Softénon. Dans ce second exemple, Sartre voit une attitude morale (ou éthique). Les parents de Liège ne témoignent pas de la volonté d'un statu quo; on n'a pas affaire à la reproduction d'un comportement qui répondrait à une explication sociologique. Au contraire, Sartre voit dans cette histoire difficile (et qu'on ne peut pas souhaiter voir répétée) un acte où des hommes et des femmes ont cherché à sortir de la pure et simple reproduction des normes sociales en vigueur et de la manière dont celles-ci peuvent se retourner contre les hommes qui les ont élaborées pour vivre, plus précisément qui les avaient élaborées comme possibilités de vivre une vie humaine. Il ne faut donc pas se précipiter à identifier les deux situations. Cherchant à réfléchir le présent dans l'écart de ces deux expériences, Sartre prend également soin d'envisager ces deux cas à rebours de la prise de position qu'on aurait pu spontanément attendre de lui. L'articulation problématique de la question de l'enfance à une conjoncture sociale et politique déterminée - celle de l'aprèsguerre en France, avec comme prisme la guerre d'Algérie - rend ainsi possible une réflexivité intellectuelle dont nous chercherons ici à préciser les conditions de possibilité et les enjeux.

Pendant ces années 1963-1965, la question de l'enfance, qui était déjà présente chez Sartre dans les années $1950^{13}$, prend une place stratégique

\footnotetext{
${ }^{12} \mathrm{~S}$ 'agissant du premier terme de l'alternative, la mort, Sartre désigne par le terme de « sacrifice » le fait que la mort de ces enfants-monstres apparaît comme la réclamation d'un avenir où l'homme produira l'homme (et pas le sous-homme), où les techniques de la procréation donneront à tous les enfants d'égales chances de vivre une vie véritablement humaine. Voir : Sartre (2015: 43).

${ }^{13}$ On connaît les phrases de Questions de méthode sur le rôle décisif de l'enfance : «L'existentialisme aidé de la psychanalyse ne peut étudier aujourd'hui que des situations où l'homme s'est perdu lui-même dès l'enfance car il n'y en a pas d'autres
} 
décisive. Les remarques sur les enfants Softénon se situent entre la publication des Mots (1963), où Sartre démonte la mystification d'une enfance qui se vit comme exceptionnelle, enfance d'un homme illustre, et la rédaction de la biographie de Flaubert que Sartre entreprend, à partir de 1966, non seulement à partir d'une enfance singulière (ce qu'il faisait déjà en 1957 dans Questions de méthode), mais comme un «enfant arriéré » (Sartre, 1987: 96) qui a un rapport particulier au langage et à la communication. À cet égard, l'entretien sur l'anthropologie de février 1966 est très instructif. L'entretien commence par une référence de Sartre à un numéro de la revue Esprit sur «L'enfance handicapée » (novembre 1965).

Dans le numéro d'Esprit, consacré à l'enfance handicapée, il y a un accord complet des médecins, analystes ou pas, sur le fait que l'erreur jusqu'à ces vingt-cinq dernières années a été de prendre l'enfant débile pour un objet, de considérer qu'il avait une lacune. [...] La seule manière est, maintenant, de traiter l'enfant comme sujet $[\ldots]$ non comme un objet qui s'insère dans la société mais comme processus sujet en cours de développement, qui change, historique, qui se trouve inséré dans un projet général et qui est en même temps une subjectivité. (Sartre, 1987 : 96)

Cette référence au numéro de la revue Esprit permet non seulement de préciser la genèse de L'Idiot de la famille, où le petit Gustave est présenté comme un enfant arriéré, mais surtout de mieux comprendre le contexte dans lequel Sartre s'intéresse au Softénon dans un dialogue qu'il entretient durant une dizaine d'années avec la revue Esprit. L'introduction du volume par Jean-Marie Domenach, qui se limite à 4 feuillets, fait deux références appuyées au « procès de Liège », puis à « la petite fille de Liège », inscrivant le propos sur l'enfance handicapée dans la suite de la polémique de l'automne 1962 (Domenach, 1965 : 578, 580).

La relation de «proximité distante » de Sartre à l'égard de la revue Esprit se fait (là aussi) sur un double plan : un premier plan politique, et même ultrapolitique, déterminé par l'engagement commun pour l'indépendance de

dans une société fondée sur l'exploitation » (Sartre, 1986 : 63) ; «Le donné que nous dépassons à tout instant, par le simple fait de le vivre, ne se réduit pas aux conditions matérielles de notre existence, il faut y faire rentrer, je l'ai dit, notre propre enfance » (Sartre, 1986 : 93). 
l'Algérie et le soutien à la lutte armée du FLN (c'est dans Esprit, par exemple, que Fanon publie ses premiers textes sur le racisme et sur l'exploitation de la main-d'œuvre nord-africaine en France métropolitaine ${ }^{14}$ ); un second plan qu'on pourrait dire moral, ou infra-politique, qui est celui des rapports aux autres et à soi, tels qu'ils peuvent être saisis au niveau des rapports sociaux qui correspondent à une médicalisation généralisée de la société. Au cours des années 1950 et 1960, la revue Esprit consacre plusieurs numéros à ce second plan d'investigation : à propos du pouvoir psychiatrique en 1952, de la prise en charge de la vieillesse et du vieillissement en 1963, ou encore, comme on l'a vu, de l'enfance handicapée en $1965^{15}$.

En quoi cette préoccupation d'Esprit pour le rapport médical est-elle importante pour Sartre ? On peut donner d'abord une réponse conjoncturelle. La période 1963-1965 suit immédiatement la signature des Accords d'Évian et la fin de la guerre d'Algérie (mars-juillet 1962). Dans ce contexte, on peut penser que la question médicale constitue pour Sartre un analyseur social et politique qui n'évalue plus la société française en priorité à partir de ses relations (de politique) «extérieures » (son système colonial), mais à partir de ses relations « intérieures », à partir de la manière dont la société française, à travers ses techniques médicales, prend en charge ses « fous », ses « enfants » ou ses « vieux ». Plus avant, l'effondrement du rapport colonial, ainsi que de l'idéologie civilisatrice européenne qui l'accompagnait, déjà gravement mise en cause par la Seconde Guerre mondiale, met en question l'ensemble des rapports sociaux au sein de la société française. Dans ce cas, le rapport médecin-patient ou la manière dont la vie est insérée dans une série de techniques médicales n'apparait plus comme un dérivé d'une détermination sociale fondamentale, comme lorsque Fanon décrivait le «syndrome nordafricain » des travailleurs immigrés nord-africains comme la conséquence pathologique d'une vie sans relations (amoureuses) et sans inscription

\footnotetext{
${ }^{14}$ Voir Fanon (1951 et 1952). Il convient aussi de rappeler l'implication de Francis Jeanson dans la revue Esprit (en parallèle avec son implication dans Les Temps Modernes), dans laquelle il dénonce la colonisation de l'Algérie dès 1950. Voir, sur ce point, Ulloa (2012). Pour le dossier complet de l'engagement d'Esprit dans la guerre d'Algérie, voir Roman (2002).

15 « Misère de la psychiatrie », décembre 1952 ; «Vieillesse et vieillissement », mai 1963 ; «L'enfance handicapée », novembre 1965.
} 
collective (Fanon, 1952) ${ }^{16}$. Le rapport médecin-malade apparaît comme le lieu d'exercice d'un certain rapport à la vie et à mort et comme une certaine manière de considérer, dans toute leur intensité, et dans leurs contradictions, les rapports sociaux que ces rapports à la vie et à la mort articulent.

Cette réponse conjoncturelle s'appuie sur une attention de longue date de Sartre pour la manière dont la médicalisation de la société était discutée au sein d'Esprit. En 1957, déjà, Sartre faisait référence à un numéro récent de la revue Esprit, intitulé Les médecins parlent de la médecine. Moquée par les communistes dans Les Lettres françaises, qui ne voyaient dans le rapport médecin-patient qu'une détermination sociale secondaire (par opposition aux rapports économiques), l'entreprise de la revue Esprit recevait un soutien inattendu (resté inaperçu en tout cas depuis 60 ans) dans Questions de méthode, en octobre-novembre 1957. Sartre y soutient, en effet, dans une très longue note, que la relation du médecin avec le malade est un «lien spécifique et profond », une «relation humaine » ou encore une « relation de personne à personne » (Sartre, 2005a : 96, note) qui instruit sur la manière dont le monde, certes avec ses conditions économiques qui sont celles d'une société capitaliste, est vécu par les hommes et les femmes depuis l'enfance dans le rapport à leur corps et dans le rapport à leur mort (Sartre, 2005a : 95). Plus généralement encore, Sartre soutient que, si c'est la société qui « décide de ses malades et de ses morts » et, dans ce cadre, que c'est « la maladie qui crée le médecin », qui est donc relatif à l'organisation socio-économique, cela n'empêche pas que la relation médicale est « une certaine manifestation - particulièrement urgente de la vie matérielle, des besoins et de la mort » (Sartre, 2005a : 96, note). La relation du médecin à ses malades est marquée, d'une part, objectivement, par une situation de souffrance, de danger et/ou d'appel à l'aide et, d'autre part, subjectivement, par une relation de nécessaire «confiance» (ibid.). Sartre en

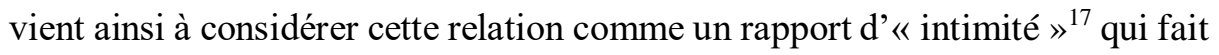
du médecin et de son patient un « couple indissoluble » qui lui apparaît (je vais

${ }^{16}$ Il en va de même de la fameuse «impulsivité criminelle du Nord-Africain », dont Les Damnés de la terre font une critique définitive en en faisant le retournement contre soi d'une violence subie mais impossible à exprimer (Fanon, 2011).

${ }^{17}$ Sartre parle aussi d'un « corps à corps » entre le médecin et le malade. 
éclairer ce point un peu plus loin) «plus intime encore que le rapport sexuel (ibid.) ». Je cite Sartre :

Ce rapport social et matériel [la relation du médecin au malade] s'affirme dans la pratique comme une liaison beaucoup plus intime encore que l'acte sexuel ; mais cette intimité ne se réalise que par des activités et techniques précises et originales engageant l'une et l'autre personne. (Ibid.)

C'est peut-être cette relation de confiance qui transparaît dans le choix du docteur Casters d'aider les parents Vandeput à mettre un terme aux jours de leur enfant. Dans le cadre de l'affaire du Softénon, le médecin de famille est à l'évidence sorti du protocole médical auquel l'équipe hospitalière ne voulait pour sa part aucunement déroger. Mais, d'où vient cette proposition qui peut sembler déroutante ou, à tout le moins, précipitée que la relation du médecin au malade est pour ainsi dire la plus intime possible?

\section{Les attitudes devant la vie et devant la mort : Sartre, lecteur d'Ariès}

La réponse apparaît en filigrane dans 2 des textes commentés ici, Questions de méthode et Les racines de l'éthique. Mais elle est divulguée par Sartre dans Qu'est-ce que la subjectivité ? (Sartre, 2013), la première conférence que Sartre a faite à l'Institut Gramsci de Rome en décembre 1961 : il s'agit du travail de Philippe Ariès sur les variations des « Attitudes devant la vie et devant la mort du XVII ${ }^{\mathrm{e}}$ au XIX ${ }^{\mathrm{e}}$ siècle» $\left(\right.$ Ariès, 1949) ${ }^{18}$ que l'historien publie dans la revue Population en 1949.

... un historien français, de droite d'ailleurs, $[\ldots]$ Ariès $[\ldots]$ a constaté ce fait extrêmement intéressant, à savoir que tout ce qui était subjectif avec une idéologie du naturel au XVIII ${ }^{\mathrm{e}}$ siècle, par exemple, donc sur le plan de la natalité : on fait les enfants sans aucun birth control et on compte sur la mort pour les ôter, [...] est devenu, au contraire, au XIX ${ }^{\mathrm{e}}$ siècle, avec toutes les pratiques bourgeoises de birth control, une opération de la personne sur son propre corps, y compris dans le domaine de la sexualité, parce que la personne

\footnotetext{
${ }^{18}$ Cet article propose un résumé de l'Histoire des populations françaises qu'Ariès avait publié l'année précédente, en 1948 (Ariès, 1948). Il est probable que Sartre n'a pas lu cet ouvrage, mais seulement le « résumé » qu'en donne l'article de la revue Population.
} 
saisit à ce moment-là son propre corps comme un objet. (Ariès, 1949 : 99100).

C'est notamment le rapport de maitrise et de contrôle technique du corps qui s'installe progressivement dans la société française, entre le $\mathrm{XVII}^{\mathrm{e}}$ et le $\mathrm{XIX}^{\mathrm{e}}$ siècle, qui est selon Sartre la contribution décisive d'Ariès : " l'attitude devant la mort, devant le corps à soigner, est entièrement neuve, c'est-à-dire qu'un corps est maintenant pour nous, à la fois la subjectivité que nous sommes et l'objet que nous sommes » (Ibid.). Dans Les racines de l'éthique, Sartre insiste sur le rapport entre les progrès techniques de la médecine et la restriction des naissances.

Changement d'attitude envers la mort et la vie dî aux changements de société. Les progrès de la médecine (progrès technique, industrialisation de la pharmacie) permettent à l'homme d'agir sur sa propre mort. C'est le mettre en face de la possibilité d'agir sur les naissances. Les maladies infantiles sont en régression et conséquemment les pratiques contraceptives ou abortives en progression.

Problème du Softénon: c'est l'antiphysis (action d'un produit pharmaceutique) qui a fait des monstres de ces enfants. En conséquence c'est l'antiphysis qui doit régler le problème (contraception ou infanticide). Le sociologue fera remarquer que l'affaire du Softénon a pour principal intérêt d'avoir fait émerger le problème au niveau de la conscience morale, mais il fera remarquer que, depuis longtemps - en France en tout cas -, certains accoucheurs ont l'habitude d'étouffer les monstres qui viennent au monde et de déclarer ensuite qu'ils sont mort-nés. Avec ou sans la complicité tacite de la mère. (Sartre, $2015:$ 28-29)

Avant 1961, des raisons politiques, qui sont évoquées par Sartre, l'ont certainement empêché de citer Ariès, dont il connaissait probablement le travail depuis longtemps ${ }^{19}$. Maurrassien, proche de l'Action française, catholique,

\footnotetext{
${ }^{19}$ On peut penser que Sartre connaissait le texte d'Ariès depuis le début des années 1950. L'exemple que Sartre prend dans Qu'est-ce que la subjectivité ? (Sartre, 2013 : 100), celui des pionniers de l'alpinisme qui traitent désormais leur corps comme un objet afin de réussir à gravir l'Everest ou l'Annapurna, est déjà utilisé par Sartre, en 1955, dans un texte préparatoire aux Mots. Voir Sartre, 2010b : 928 : « On ne fait rien du corps sans techniques nouvelles. Il faut une nouvelle médecine (elle commence. Himalaya) et de nouvelles tâches (le mur du son dépassé) ».
} 
monarchiste, Ariès était aussi un défenseur de l'Algérie française. Ce sont probablement aussi des raisons politiques qui ont rendu possible que Sartre cite Ariès dans la discussion qui suit sa conférence à Rome en décembre 1961. Malgré ses positions politiques et sa sympathie pour l'OAS, Ariès, encouragé notamment par un de ses amis, le peintre polonais Joseph Czapski, dénonce la répression sanglante de la manifestation que le FLN organise à Paris le 17 octobre 1961, ainsi que la pratique de la torture par l'armée française ${ }^{20}$. Il écrit un article dans La Nation française intitulé «Le racisme dans notre société industrielle ${ }^{21} \gg$, qui lui vaudra la reconnaissance de Pierre Vidal-Naquet. Ariès est dès lors un allié objectif de Sartre dans le combat pour l'indépendance de l'Algérie. Ou, plus précisément encore, Sartre, très sensible aux courants sociaux, notamment médiatiques, en bon élève des durkheimiens, ne peut manquer de formuler ses positions d'une manière qui puisse aussi toucher les personnes qui ne sont pas de son bord politique ${ }^{22}$. La citation assumée d'Ariès ne peut être comprise que dans ce contexte précis de la guerre d'Algérie.

Dans son article sur les «Attitudes devant la vie et devant la mort », Ariès s'intéresse à la révolution démographique que la France connaît au XIX ${ }^{\mathrm{e}}$ siècle parallèlement à la révolution industrielle. Ce qui frappe Ariès, c'est la façon dont le développement de la médecine, qui a permis de retarder l'advenue de la mort, a progressivement impliqué un souci pour la maîtrise des naissances et pour les moyens de contraception : «la révolution de la mort a précédé celle de la vie. » (Ariès, 1949 : 467). On ne peut penser agir sur la génération et sur les naissances que si la vie (et la mort) est devenu un «terrain d'intervention » (Ariès, 1960b : 325). Ce faisant, ce qui intéresse plus précisément Ariès, c'est la manière dont «l'humanité outillée » (celle de la révolution industrielle et

${ }^{20}$ Cf. Gros, 2013. La répression policière lors de la manifestation du 17 octobre 1961 a été une des plus importantes de l'histoire récente de l'Europe occidentale. Elle a fait (probablement) plus de 100 morts. Voir Péju et Péju, 2011.

${ }^{21}$ Ariès, 1961 : «Parisiens, mes amis, nous devons plutôt faire appel à la pitié et même à la justice, pour ces hôtes qui assurent chez nous les tâches humbles et méprisées, victimes des ruses de la politique, de l'indifférence de la société ». Ariès enfoncera le clou en janvier 1962 en intervenant directement sur la question de la torture et sur sa propre complaisance jusque-là envers son usage par les forces armées françaises.

${ }^{22}$ Comme le montre l'intervention de Sartre lors d'un des grands procès de la guerre d'Algérie, le procès Ben Sadok, auquel il participe en décembre 1957. Voir, sur ce point, Bourgault et Cormann (2014). 
technique) du XIX ${ }^{\mathrm{e}}$ siècle, par opposition à « l'humanité fataliste » des sociétés antérieures, en vient à «s'appliquer elle-même les nouvelles techniques scientifiques » (Ariès, 1949 : 464).

Mais, a-t-on bien réfléchi à ce passage d'une humanité coutumière et résignée, à une humanité outillée, et déterminée à s'appliquer elle-même les nouvelles techniques scientifiques ? C'est, sans conteste, un des phénomènes les plus considérables de l'histoire contemporaine, moins encore par ses conséquences que par l'intensité du changement imposé au comportement psychologique. C'est l'interprétation psychologique de la révolution démographique dont il convient de signaler ici l'intérêt, pour le démographe sans doute, mais aussi pour le sociologue et l'historien, trop souvent fermés à cet ordre de phénomène. Il y a eu changement dans l'attitude fondamentale de l'homme devant l'existence, devant la vie et la mort. (Ibid.)

L'humanité en vient à appliquer à son propre corps la nouvelle maîtrise technique qu'elle a forgée dans son rapport au monde. La diffusion massive de la contraception apparaît ainsi à Ariès comme une invention récente qui ne remonte guère au-delà de 1890 (Ariès, 1949 : 469). Pour le dire d'une formule, ce qui intéresse donc Ariès, c'est la manière dont les techniques scientifiques deviennent des techniques du corps ${ }^{23}$. Dès lors, son travail s'inscrit dans une double continuité théorique : d'une part, celle à laquelle on l'associe souvent, l'histoire des mentalités de l'École des Annales, d'autre part, celle de l'École française de sociologie, qu'évoque sans conteste la référence au concept maussien de techniques du corps. L'histoire des attitudes devant la vie relève par conséquent d'une audacieuse synthèse méthodologique.

${ }^{23}$ « C'est par l'action sur la naissance et la mort que la technicité propre à notre époque a cessé d'être périphérique à l'homme, pour pénétrer profondément son comportement, phénomène qui est devenu l'un des faits les plus importants de l'histoire contemporaine. » (Ariès, 1949 : 468). 


\subsection{Ariès et Lucien Febvre : une histoire des sensibilités face à la vie et à la mort}

Ariès fait de l'histoire des mentalités. Il appartient donc à l'École des Annales. La démonstration semble ne pas devoir être faite. Lorsqu'il publie son histoire de l'enfance (Ariès, 1960a), l'ouvrage ne fait-il pas l'objet d'un compte rendu élogieux dans les Annales (Flandrin, 1964) ? Et Peter Gay ne présente-t-il pas le même livre comme les «Annales of childhood » dans un autre compte rendu qui a fait date aux États-Unis (Gay, 1963 ; Gros, 2006) ? L'histoire semble d'autant plus entendue qu'Ariès a lui-même très tôt reconnu l'importance de Bloch et de Febvre pour sa formation d'historien ${ }^{24}$. Pourtant, à s'en tenir là, la relation entre Ariès et les Annales peut apparaître assez superficielle. Ainsi André Bruguière ne consacre-t-il, dans son histoire intellectuelle des Annales, que quelques remarques à l'auteur de L'enfant et la vie familiale sous l'ancien régime (Burguière, 2006). Ariès lui-même brouille quelque peu les pistes. En effet, si dans Le temps de l'histoire, en 1954, il reconnaît l'influence décisive des deux maîtres des Annales, c'est surtout pour mettre l'accent sur Marc Bloch dont il regrette que la mort en martyr pendant la guerre a empêché de produire l'œuvre théorique à la mesure de son talent d'historien (Ariès, 1954a : 292). Il laisse ainsi dans l'ombre l'importance pour son histoire des attitudes devant la vie et dans la mort les « idées sur l'histoire » de Lucien Febvre (ibid. : note 1$)^{25}$.

Pourtant, c'est bien aux essais méthodologiques que Febvre consacre, dans la seconde moitié des années 1930, au projet d'élaborer une histoire des sensibilités qu'Ariès doit l'élan de son travail. L'idée d'une histoire des attitudes devant la vie et devant la mort ne se rattache pas à l'histoire des mentalités en général ; elle trouve son impulsion dans les articles critiques et les réflexions sur l'histoire que Febvre développe dans le cadre - et en marge de l'Encyclopédie française, l'entreprise collective et interdisciplinaire qui fait tout au long de ces années 1930 le bilan des derniers développements scientifiques et dont l'historien a la responsabilité générale. Dans l'article

\footnotetext{
${ }^{24}$ Ariès, 1954 (le chapitre est daté de 1949).

${ }^{25}$ Dans cette note de bas de page, Ariès signale, pour ne rien en dire, la publication récente, en 1952, des Combats sur l'histoire qui rassemblent «les articles de critique où ses idées sur l'histoire [celles de Febvre] sont le plus développées ». Voir Febvre, 1995a.
} 
« Psychologie et histoire » qu'il donne au volume VIII de l'Encyclopédie, en 1938, Febvre met en exergue les différences de mentalités envers la vie et envers la mort qu'on peut observer entre les sociétés modernes, les sociétés médiévales et les sociétés primitives ${ }^{26}$. S'appuyant sur Frazer et sur Huizinga, mais peut-être surtout sur l'Histoire littéraire du sentiment religieux en France d'Henri Bremond, Febvre fait remarquer que les attitudes des sociétés humaines face à la vie et à la mort ne peuvent se résumer à la tautologie d'un instinct de conservation, d'une vie attachée à elle-même, d'une vie soucieuse de la vie (et de la mort). Il regrette aussi que les historiens n'aient pas encore pris soin de réunir les données susceptibles d'éclairer cette enquête (Febvre, 2009a: 186-187). En renvoyant à son article de 1925 sur «La première renaissance française » (Febvre, 2009b : 783-784) - une très belle contribution à une histoire des idées bourgeoises, que le jeune Sartre a aussi pu connaître -, Febvre attire l'attention sur l'intérêt des matériaux iconographiques pour l'historien, comme il y insiste aussi dans un grand article de la même période, «La sensibilité et l'histoire » (Febvre, 2009c : 201) ${ }^{27}$.

Au-delà des références anthropologiques et historiques, Febvre renvoie systématiquement ses réflexions de méthode aux travaux de psychologie de son ami Henri Wallon. Tous deux professeurs au Collège de France, Febvre et Wallon étaient alors amis depuis près de quarante ans, depuis les années passées ensemble à l'École Normale Supérieure et à la Fondation Thiers. Le socialiste Lucien Febvre n'approuvait certes pas les idées communistes de son ami, mais cela ne l'empêchait de prendre très au sérieux ses travaux et de reconnaître également la valeur des recherches d'inspiration marxiste. Wallon, à qui Febvre a confié le volume sur La vie mentale, apparaît ainsi comme le principal aiguillon des «idées » de Febvre sur l'histoire ${ }^{28}$. Rendant compte, en 1935, du volume À la lumière du marxisme, qui résumait les perspectives marxistes sur les sciences et les techniques, et qui était dirigé par Wallon, Febvre relevait 3 considérations principales dans l'approche matérialiste de la technique

\footnotetext{
${ }^{26}$ Febvre, 1938. Repris sous le titre «Une vue d'ensemble. Histoire et psychologie » (Febvre, 1995b ; 2009a).

27 À la suite d'Émile Mâle, Febvre insiste en particulier sur l'étude des costumes. L'étude des costumes constituera précisément le point de départ de l'histoire de l'enfance d'Ariès.

${ }^{28}$ Febvre, 2009a : 190 ; 2009c : 193-194.
} 
proposée par le psychologue : premièrement, le développement des techniques (industrie automobile et aéronautique, télécommunications et mass médias) modifie nos «façons de percevoir, de sentir et de réagir »; deuxièmement, ces développements techniques qui paraissent déstabiliser les «capacités de notre organisme » mettent en question les représentations qu'on pouvait se faire jusque-là des structures de l'expérience humaine et de notre être-au-monde (comme la physique moderne renouvelle notre connaissance de la structure du monde) ; troisièmement, Wallon soutient la nécessité d'études historiques capables d'établir le rapport précis entre «le système général des idées et les techniques générales de la vie $\gg^{29}$.

De ces échanges avec Wallon, Febvre retient en particulier l'encouragement du psychologue marxiste à promouvoir l'étude des différences de mentalité qui ont été produites par le développement des techniques : d'un côté, pour les périodes anciennes, l'historien ne peut pas ignorer que l'humanité était alors privée des développements techniques modernes et devaient dès lors faire face à la nature avec « sa seule force musculaire » (Febvre, 2009a : 190); de l'autre, pour la période contemporaine, Wallon insiste sur les effets rapides des développements techniques sur les hommes, sur leurs manières de percevoir le monde comme sur leurs manières d'agir sur leurs $\operatorname{corps}^{30}$. C'est aussi cette discussion de Febvre avec Wallon qui a placé sur la route d'Ariès le travail de sociologie du corps de Marcel Mauss.

\footnotetext{
${ }^{29}$ Voir Febvre, 2009d : 853-854, y compris la note 1 de la p. 854. Febvre rend compte de l'ouvrage À la lumière du marxisme publié en 1935 aux Éditions sociales internationales ([Collectif], 1935).

${ }^{30}$ Voir aussi, à ce sujet, "L'enquête continue », la contribution de Febvre à un autre tome de l'Encyclopédie française consacré aux Arts et Littératures (Febvre, 2009e : 49) : « Tout ceci dans un monde complètement bouleversé et rénové par un prodigieux ensemble de découvertes scientifiques aboutissant à la télégraphie sans fil, à l'aviation, au phonographe, à la radio - demain, à la télévision, etc. Tout ceci, tellement fort et profond qu'hier Henri Wallon nous en avertissait: déjà, en si peu d'années, ces inventions d'une prodigieuse universalité ont commencé d'agir sur nos organismes, de modifier nos perceptions, de transformer une humanité depuis des siècles et des siècles à peu près immobile dans sa constitution. »
} 


\subsection{Ariès et Marcel Mauss : les techniques du corps, de la sociologie à la psychologie}

Par l'insistance sur le concept de technique du corps, l'entreprise de Philippe Ariès est en effet également redevable à l'égard du célèbre article de Mauss et à la collaboration que celui-ci souhaite également nouer avec les psychologues (Mauss, 1950) $^{31}$. En 1934, devant la Société de psychologie, Mauss attire, on le sait, l'attention sur le fait qu'à côté des techniques outillées ou «avec instruments », il existe une autre sorte de techniques, qui sont sans instruments : les techniques du corps. Il y a, soutient Mauss, une éducation nécessaire du corps, ou encore un dressage du corps. Selon lui, il n'y a rien de naturel dans le corps, il n'y a que des manières particulières d'en user, socialement déterminées et acquises en fonction d'une certaine valeur (efficacité ou prestige). Chez Mauss, la notion de technique du corps désigne par conséquent l'ensemble des manières d'utiliser notre corps pour marcher, nager, respirer, accoucher, se reposer ou dormir, qui font aussi l'objet, comme les techniques outillées, d'une transmission sociale et sont donc, elles aussi, variables d'une société à une autre. Cependant, Ariès déplace le propos de Mauss : il ne s'intéresse pas aux techniques du corps invisibilisées qui travaillent sous les techniques instrumentées, à titre, pourrait-on dire, de conditions de possibilité transcendantale de notre action dans le monde. On dirait volontiers qu'il s'intéresse, comme à l'inverse, aux répercussions du développement technique sur les techniques appliquées au corps humain. Mais on peut aussi bien considérer que, ce faisant, Ariès approfondit la perspective maussienne sur deux points. Premièrement, il comble un point aveugle de l'article de Mauss qui ne s'intéresse pas à la vie et à la mort en tant que telles et saisit l'être humain, disons, de l'enfance à la vieillesse ${ }^{32}$. Dans la description des techniques du corps selon les âges de la vie, Mauss, en sociologue, inscrit les usages du

\footnotetext{
${ }^{31}$ À ma connaissance, il n'existe pas d'étude sur la relation d'Ariès à Mauss. Le présent dossier me fait considérer que cette lecture a eu lieu et qu'elle a été décisive dans l'élaboration de son travail. Anna Iuso (Iuso, 2010) a suggéré l'importance de l'article de Mauss sur le concept de personne pour comprendre la genèse du travail d'Ariès sur le sentiment d'enfance.

${ }^{32}$ On remarquera que « De l'enfance à la vieillesse » est le sous-titre du tome VIII de l'Encyclopédie française consacré à la vie mentale que nous avons évoqué dans la section précédente.
} 
corps entre les pratiques de seconde naissance ou de naissance sociale, telles que les pratiques d'exposition, d'une part, et la vieillesse, d'autre part. Ni la vie ni la mort (pas même les phénomènes de mort sociale) ne sont prises en considération par Mauss. Cela est certainement dû au matériau anthropologique sur lequel Mauss appuie le catalogage des techniques du corps. Les effets du développement technologique sur les individus ne peuvent pas y trouver leur place. Par méthode, il était même probablement nécessaire à la «démonstration» de Mauss de distinguer strictement les deux registres techniques. Il y a des techniques du corps et il y a des technologies du corps. Mais, deuxièmement, le travail d'Ariès porte profondément la marque du repérage maussien : Mauss avait en effet bien montré, par le truchement des techniques du corps, qu'il y avait, à côté de l'ordre de la contrainte sociale, un ensemble de moyens par lequel la société s'impose à l'individu, non pas de l'extérieur, mais de l'intérieur. Ariès reprend ce geste à son compte, mais afin de dégager cette normativité immanente non pas objectivement, à savoir à travers la disposition sociale du corps vers l'action, mais cette fois subjectivement ou psychologiquement, de telle sorte que l'action de l'humanité sur les corps doive aussi être considérée selon ses effets dans l'ordre de la pensée, en particulier dans la manière dont l'humanité se représente elle-même.

Par suite, l'histoire des attitudes devant la vie et devant la mort qu'Ariès élabore à partir du projet febvrien d'une histoire des sensibilités présente plusieurs analogies avec la psychologie des émotions que Sartre écrit dans le même contexte intellectuel de la seconde moitié des années 1930 : celui de la collaboration nécessaire, dans une période de transformations sociales majeures et de crise de la civilisation européenne, de la psychologie, de la sociologie et de l'histoire.

\subsection{Sartre et Ariès : les techniques du corps, d'une psychologie des émotions à une réflexion sur l'histoire}

Dans l'Esquisse d'une théorie des émotions (Sartre, 1939), Sartre propose une synthèse des travaux psychologiques de l'époque afin de contester l'appréciation négative qu'on a le plus souvent des réactions émotionnelles : celle-ci serait au fond une perturbation organique qu'il s'agirait de contrôler afin d'éviter de produire des comportements inadaptés à ce que le contexte 
requiert comme réponse rationnelle et adaptée. Pour Sartre, au contraire, les émotions ne sont rien d'autre que la manière dont la conscience cherche, en agissant sur son propre corps, à rester concernée par la situation dans laquelle elle se trouve, malgré l'impossibilité que le monde lui oppose de continuer à agir selon les moyens pragmatiques (Mauss dirait : selon les techniques) qu'elle utilisait jusque-là (Cormann, 2012). À cet égard, l'émotion chez Sartre peut, à bon droit, être considérée comme « une technique du corps » : elle consiste bien à agir sur son corps de façon à agir sur le monde sans ustensile, sans instrument. À l'instar de Mauss, Sartre dégage, sous les techniques instrumentées, des techniques du corps qui constituent en quelque sorte la condition de possibilité des premières. La conscience pragmatique est soutenue par une conscience émotionnelle qui situe ses conduites dans un monde humain.

La théorie phénoménologique des émotions de 1939 s'inscrit très clairement dans la continuité de l'Encyclopédie française, dirigé par Febvre, et de la collaboration prolongée de Mauss avec la Société de psychologie. Son attention à la question du corps et des émotions, comprises comme des techniques du corps, relève cependant uniquement d'une anthropologie phénoménologique ; rien alors ne renvoie à une histoire des attitudes devant la vie et devant la mort, à une histoire des émotions ou des techniques du corps. C'est l'apport déterminant de l'étude d'Ariès pour Sartre. Elle donne une autre portée à l'article de Mauss sur les techniques du corps : elle permet de passer de la psychologie à l'histoire. Ou plutôt, dans la perspective inaugurée par Febvre, elle permet de considérer la psychologie, non seulement comme un objet légitime d'étude historique, mais comme une approche méthodologique pertinente pour l'histoire et l'historien.

La conférence de Rome de 1964 en fournit une mise en œuvre originale qui est ajustée à la compréhension du procès d'infanticide de Liège et des réactions qui ont été suscitées par l'acquittement de la famille Vandeput. Il s'agit de comprendre comment les effets de certains développements technologiques et médicaux désarçonnent les habitudes mentales qui relèvent d'un autre rapport au monde et à l'existence qui n'était pas marqué par une médicalisation généralisée. Sartre s'attache donc en quelque sorte à réécrire l'article d'Ariès sur les «Attitudes devant la vie et devant la mort » en 1963 ou 1964. 


\section{L'analyse de l'affaire du Softénon selon Sartre}

Cela nous donne l'occasion de revenir sur la manière dont Sartre envisage l'affaire du Softénon. L'analyse de Sartre assume en effet un double décalage par rapport au travail pionnier d'Ariès. En premier lieu, le Softénon oblige Sartre à déplacer la problématique d'Ariès : celui-ci étudie l'effet que la possibilité d'agir sur la mort a eu sur la manière d'agir sur la naissance. Avec le cas du Softénon, on se trouve porté, au fond, à une étape postérieure des interactions entre les attitudes devant la vie et devant la mort : ce que met en évidence l'affaire du Softénon, ce n'est pas la manière dont l'action sur la mort autorise des pratiques d'avortement ou d'infanticide, mais la manière dont les technologies de la gestation et de la naissance qui produisent des vies inhumaines exposent l'humanité à pratiquer l'avortement ou l'infanticide. La mort maîtrisée et retardée n'impose donc pas ici un contrôle et une limitation de la vie ; ce qui impose une limitation de la vie, c'est le fait d'une « technique mal appliquée » (Sartre, 2015 : 44), c'est le fait de produire techniquement, médicalement, une mort-dans-la-vie, c'est-à-dire une existence qui se referme immédiatement sur la personne qui la vit. En second lieu, la perception sociale du procès d'infanticide est l'occasion pour Sartre d'interroger la notion même d'histoire. Par la précision biopolitique dont il fait preuve, Sartre cherche à conjurer le positivisme sociologique auquel l'histoire peut faire allégeance. On a rappelé que l'objectif de Sartre était de compliquer l'interprétation sociologique qui était donnée de l'infanticide de Liège, et qui constituait le fond des commentaires produits dans Esprit et dans Les Temps Modernes. Dans Les racines de l'éthique, il ne s'agit pas pour lui de contester frontalement la validité des données sociologiques. L'infanticide de Liège s'inscrit bien dans les possibilités d'une société donnée, avec ses techniques de contrôle des naissances, ses normes bourgeoises, par exemple un certain rapport de l'enfant à ses parents. Toutefois, Sartre se refuse à y voir la confirmation explicite (on pourrait dire «éclatante») de mœurs sociales qui auraient été jusque-là pratiquées en secret et revendiqueraient aujourd'hui d'être pratiquées en pleine lumière. La position de Sartre est à l'opposé : il s'agit pour lui de reconnaître un acte qui ne revendique aucune forme d'exemplarité, qui ne cherche certainement pas à être répété, mais qui cherche au contraire à ne pas être répété, à ne pas devoir être répété, un acte qui vise l'impossibilisation de sa répétition. 
Cela signifie que l'acte des infanticides de Liège ne peut aucunement devenir l'exemple premier d'une pratique répétitive (fondée sur une valeur, un impératif) ni d'une loi (systématisation de la pratique). Pourtant la structure de cette conduite est normative. Cette conduite s'est donnée à la fois et dès le départ comme ayant à être tenue (il y a eu débat, hésitation, crise intérieure et décision) et comme conduite spécifique et singulière dépendant de circonstances datées, bref comme une conduite unique et sans retour prévisible, comme conduite historique et non répétitive. (Sartre, $2015: 42)^{33}$

À certains égards, ce que Sartre reproche aux sociologues, c'est de ne pas faire assez de sociologie et de se contenter paresseusement d'un peu de (mauvaise) histoire, de se contenter d'inscrire les pratiques d'aujourd'hui dans la continuité des pratiques d'autrefois, fors la mauvaise foi dans lesquelles ces dernières étaient réalisées. C'est négliger, on l'a déjà rappelé, que l'infanticide de Liège s'inscrit dans un cadre biopolitique : les hommes cherchent à modifier leur rapport à leur existence, mais ils n'y parviennent (aujourd'hui) qu'imparfaitement. Le recours à l'histoire ne peut donc pas consister à naturaliser un comportement en le projetant dans le passé. À l'inverse, l'attention aux technologies de la vie et de la mort impose de prendre en compte une historicité fondamentale de l'existence humaine, une part d'imprévisibilité dans la manière dont ces technologies de l'existence vont refluer sur l'existence elle-même. L'histoire oblige dès lors à soutenir qu'on ne peut jamais savoir de quoi l'avenir sera fait : «Sans doute l'antiphysis est elle-même l'histoire [...] : on ne sait jamais exactement ce qu'on fait, aujourd'hui, quand on prescrit un médicament » (Sartre, 2015 : 44). Mais, plus fondamentalement encore, ce que le positivisme sociologique recouvre, c'est le fait même de l'histoire, le fait que l'humanité s'affronte à chaque instant à la possibilité d'être autre que ce qu'elle est à ce moment déterminé : " possibilité historique, c'est-à-dire [...] la possibilité de notre espèce et non de notre temps » (Sartre, 2015 : 40).

${ }^{33}$ Ainsi le travail d'Ariès permet-il d'éclairer la volte-face argumentative de Sartre lorsqu'il dégage la maxime négative de l'infanticide de Liège : «Je tue aujourd'hui mon enfant pour que demain aucune mère ne soit tentée de tuer le sien » (Sartre, 2015 : 44). 
La possibilité de penser la possibilité historique se présente donc comme l'enjeu fondamental de la présence insistante chez Sartre du travail de Philippe Ariès. Son intérêt pour les émotions et pour le corps l'a rendu attentif à l'histoire des sensibilités qu'Ariès trouve en gestation dans les essais critiques de Febvre. Mais les dernières lignes de l'article, qui renvoient directement au travail historien de Febvre, ont dû également retenir l'attention de Sartre. À la toute fin de son article, Ariès insiste, comme nous l'avons déjà dit, sur le fait que les techniques de contraception et de contrôle des naissances qu'il a étudiées d'un point de vue démographique sont une pratique sociale extrêmement récente, « comme le chemin de fer ou l'électricité » (Ariès, 1949 : 469). Il estime en effet qu'avant cette période une telle maîtrise de la natalité était impossible.

Contentons-nous d'insister sur cette observation : au moment où aujourd'hui, les diverses techniques anti-conceptionnelles sont devenues d'un usage courant, on est étonné quand on constate la date récente de leur usage. C'est une technique du $\mathrm{XIX}^{\mathrm{e}}$ et du $\mathrm{XX}^{\mathrm{e}}$ siècles, comme le chemin de fer et l'électricité. Le souci de restreindre les naissances était antérieur, sinon à leur découverte, du moins à leur emploi. On a peine à croire que les contraintes religieuses fussent assez fortes pour suffire à les interdire sur une si vaste échelle. Le silence de la littérature érotique à ce propos est assez significatif. Ce n'était ni ignorance, ni soumission, mais bien, si on me pardonne cet affreux néologisme : impensabilité. Dans un monde donné, il y a des actes impossibles, des choses impensables. Et on a vécu longtemps dans un monde d'où était exclue l'idée d'une réflexion, d'une prévision, d'une intervention qui eussent dissocié la totalité rigoureuse, quoique implicite, de l'acte sexuel. À ce monde s'est substitué un autre, le nôtre, où l'acte sexuel, comme la maladie, ne se distingue plus essentiellement des autres opérations de la nature animée ou inanimée que les techniques modifient selon un plan déterminé. Deux mondes ou deux civilisations. (Ariès, 1949 : 469-470)

Pourquoi, donc, une maîtrise des naissances était-elle impossible ? Ariès ne suit pas l'explication qui pourrait sembler la plus évidente : ce ne sont pas des raisons religieuses qui auraient interdit le contrôle de la natalité. Ariès l'affirme : si un tel contrôle n'a pas été possible, c'est parce qu'il était tout simplement impensable. Et pourquoi était-il impensable? Parce qu'il n'était pas possible avant la fin du XIX ${ }^{\mathrm{e}}$ siècle de défaire l'unité de l'acte sexuel, de 
décomposer « la totalité rigoureuse, quoique implicite, de l'acte sexuel » (ibid). En somme, la contraception massive était impossible non pas à cause d'interdictions religieuses, mais parce que les normes sociales (y compris religieuses) s'étaient faites en quelque sorte techniques du corps naturalisées, rendant impossible de considérer l'acte sexuel comme une technique du corps qui a ses moments, ses enchaînements, ses arrêts et sur laquelle il est donc possible d'agir. L'argument peut sembler rapide et déroutant. Le terme d'impensabilité renvoie cependant de façon incontestable au grand ouvrage de Lucien Febvre sur Le problème de l'incroyance au XVI siècle (Febvre, 1968).

\section{Sartre et l'histoire des sensibilités : de l'impensabilité historique à l'expérience du possible}

Dans Le problème de l'incroyance au XVI siècle. La religion de Rabelais, on le sait, Lucien Febvre conteste le fait que Rabelais ait pu être athée. Et Febvre de soutenir, déjà, que cela aurait été impossible à Rabelais d'être athée parce qu'il ne disposait pas des mots et des habitudes mentales nécessaires pour penser un tel rapport au monde. Pour lui, c'était impensable. Un écart décisif sépare cependant la perspective d'Ariès de celle de Febvre: Ariès n'accorde pas un rôle déterminant à la religion. Pour le dire simplement, une histoire des attitudes devant la vie et devant la mort doit relativiser l'influence des représentations religieuses. Par son geste, Ariès se déporte de l'approche choisie par Febvre, qui étudie non pas la différence des attitudes devant la vie, mais les «prises de la religion sur la vie » (Febvre, $1968: 307)^{34}$. Ce qui relève de l'impossible et de l'impensable, selon Ariès, ne renvoie pas à quelque chose qui ferait défaut dans les mots ou dans les idées en général, mais dans la possibilité que les sociétés humaines se représentent ou ne se représentent pas

\footnotetext{
${ }^{34}$ Chez Febvre, contrairement à ce qui se passe chez Ariès, l'histoire des sensibilités accorde un rôle central aux représentations religieuses. Le passage suivant témoigne de la dépendance de la vie - et des représentations de la vie et de la mort - à l'égard de la religion qui se dégage des études de Febvre: «Autrefois, au XVI ${ }^{\mathrm{e}}$ siècle[,] le christianisme, c'était l'air même qu'on respirait dans ce que nous nommons l'Europe et qui était la Chrétienté. C'était une atmosphère dans quoi l'homme vivait sa vie, toute sa vie - et non pas seulement sa vie intellectuelle, mais sa vie privée aux actes multiples, sa vie publique aux occupations diverses, sa vie professionnelle quel qu'en fût le cadre » (Febvre, $1968: 308)$.
} 
de pouvoir agir sur le corps humain et sur elles-mêmes. Le point n'a pas échappé à Sartre. Dans son analyse des réactions sociales face à l'affaire du Softénon, il réfute l'idée que les normes religieuses pourraient être déterminantes en dernière instance. Selon Sartre, les normes religieuses n'ont en rien touché au naturalisme éthique des sociétés paysannes (Sartre, 2015 : 2627). Il n'est donc pas étonnant que Sartre se penche sur ce qu'il appelle luimême dans la Conférence de Cornell des «techniques corporelles ${ }^{35}$.

Dans Les racines de l'éthique, Sartre ne fait pas sans raison une franche allusion à $L$ 'An $V$ de la révolution algérienne (Fanon, 1959), un ouvrage qui a marqué le philosophe et qui nous permet de revenir une dernière fois à l'articulation, dans le texte de Sartre, entre la révolution algérienne et le cas du Softénon (Sartre, 2015 : 74-75, n. 54). Dans L'An V de la révolution algérienne, en effet, Fanon décrit la manière dont la société algérienne en lutte, pourtant réputée archaïque, pourtant tournée vers les traditions musulmanes par opposition aux valeurs de la France, s'est montrée capable en 5 ans, de 1954 à 1959 , de s'approprier de nouvelles techniques de lutte et de combat, mais aussi de nouvelles formes de communication, de nouvelles modalités de cohabitation entre hommes et femmes et de nouvelles techniques du corps. Loin des descriptions qui décrivent les Algériens comme des terroristes, Fanon décrit ainsi, de façon extrêmement concrète, la création d'une nouvelle communauté ou d'une nouvelle société dans la totalité de ses dimensions constitutives.

L'exemple de l'Algérie contemporaine permet de comprendre l'intérêt de Sartre pour un autre écart qu'Ariès instaure à l'égard de l'histoire des sensibilités telle que Febvre la met en œuvre notamment dans Le problème de l'incroyance. Ariès ne cherche pas identifier une différence de mentalité entre la mentalité des hommes du $16^{\text {ème }}$ siècle et l'homme d'aujourd'hui. Ou, pour le dire encore autrement, d'un point de vue méthodologique, l'objectif de l'historien Ariès n'est pas, comme pour Febvre, de déjouer les pièges de l'anachronisme. Pour son propre compte, Ariès commence, en quelque sorte, là où Febvre s'arrête. Il cherche à cerner un changement dans les attitudes

${ }^{35}$ Voir les notes préparatoires de la conférence de Cornell, Beinecke Library, Yale University, New Haven (Notes for lecture on ethics prepared for Cornell University, holograph). John Gerassi Collection of Jean-Paul Sartre - GEN MSS 441, Box 5, folder 59, passage inédit. 
devant la vie au sein de la modernité - entre le XVII ${ }^{\text {ème }}$ et le XIX ${ }^{\text {ème }}$ siècle. C'est l'attention d'Ariès à cette différence, d'abord inaperçue, qui passe à l'intérieur même de la modernité qui intéresse Sartre et qui, selon lui, doit être radicalisée. L'intérêt de Sartre pour la médecine et pour la gouvernementalité biopolitique, dont nous sommes partis, correspond à cette vigilance de Sartre pour ce qui est en train de changer dans la société française (et dans la société occidentale) des années 1950 et 1960. La trame biographique qui y est associée autorise par ailleurs Sartre à aiguiser l'hypothèse proposée par Ariès et à instruire le dossier de ce changement social et des transformations subjectives qu'il induit depuis le XIX ${ }^{\text {ème }}$ siècle.

Qu'en est-il dès lors, chez Sartre, de la catégorie d'impossibilité ou d'impensabilité qui est la marque des débats que suscite l'ambition de Febvre et d'Ariès au sein des Annales de faire une histoire des sensibilités ? On pourrait dire qu'il s'agit pour lui de séparer les deux termes afin de faire sa place à l'expérience historique du possible. L'impensable (tel un acte d'infanticide qui est exigence d'une autre forme de vie) est pour Sartre quelque chose comme une possibilité-limite, une possibilité «toute récente», dirait Ariès, une possibilité qui est tout juste possible, autrement dit encore, l'imminence d'une possibilité. Il s'agit donc bien pour Sartre de se donner les moyens de mettre en évidence une différence dans ce qui constitue notre aujourd'hui. En réalité, on peut aisément s'y méprendre. Une possibilité, c'est cela même qui pourrait ne pas apparaître comme possibilité, mais au contraire comme la répétition d'une norme existante (qui éventuellement était jusque-là voilée). Les réactions médiatiques à l'issue du procès d'infanticide de Liège illustrent ce risque, qui ne voient dans l'attitude des parents Vandeput qu'une forme de violence pour laquelle la «mentalité bourgeoise» aurait désormais une tolérance condamnable. En particulier, avec l'affaire du Softénon, Sartre met en question la clôture éthique de la situation de l'intellectuel de gauche en 1965, alors que la fin de la Guerre d'Algérie et l'installation de logiques néocoloniales est le signe de sa clôture politique. C'est le sens même de l'autopsie qu'il entreprend dans Questions de méthode et dans Les racines de l'éthique des habitudes mentales des Temps Modernes et de la revue Esprit.

Avec le procès du Softénon, Sartre développe ainsi une histoire des sensibilités contemporaines face à la vie et face à la mort. Grâce à la lecture d'Ariès et aux propositions de méthode de Febvre, il dépasse ce qu'il reste 
d'intellectualisme dans le concept maussien de technique du corps. À l'instar d'Henri Wallon, la psychologie sartrienne est une psychologie matérialiste qui ne pouvait pas rester indifférente au développement des technologies de l'existence. Par conséquent, son analyse du procès de Liège ne se limite pas à l'identification de nouvelles techniques du corps ou à la possibilité de nouvelles formes d'expérience. Ce à quoi Sartre est confronté par l'intermédiaire de l'infanticide de Liège, c'est à l'expérience (éthique) de « l'histoire en train de se faire » (Sartre, 2015 : 42). Par les liens qu'il noue entre la vie, la politique et l'histoire, Sartre n'appartient pas seulement à l'histoire intellectuelle de l'histoire des Annales; il apporte une contribution majeure à une histoire soucieuse de mettre en question le sens que les savoirs, les scientifiques et les intellectuels donnent $\mathrm{au}(\mathrm{x})$ rapport(s) entre la vie et l'histoire.

\section{Bibliography}

[Collectif] (1935). À la lumière du marxisme, Paris: Éditions sociales internationales.

AlloA, E. (2018). « Merleau-Ponty à Madagascar. L'épreuve de l'étranger et la décolonisation de la pensée ». Chiasmi international, 19:115-126.

ARIÈs, P. (1961). «Le racisme dans notre société industrielle ». La Nation française, 25 octobre.

- (1960a). L'enfant et la vie familiale sous l'ancien régime. Paris : Plon.

- (1960b). "Interprétation pour une histoire des mentalités ». In : La Prévention des naissances dans la famille. Ses origines dans les temps modernes. Paris : Presses Universitaires de France.

- (1954a). Le temps de l'histoire. Monaco : Éditions du Rocher

- (1954b). «L'histoire existentielle». In : Le temps de l'histoire. Monaco : Éditions du Rocher, 291-311.

- (1949). «Attitudes devant la vie et devant la mort du XVII ${ }^{\mathrm{e}}$ au XIX ${ }^{\mathrm{e}}$ siècle. Quelques aspects de leurs variations ». Population, 3 : 463-470.

- (1948). Histoire des populations françaises et de leurs attitudes devant la vie depuis le XVIIIe siècle. Paris : Self.

Bourgault J. et G. CORMANN (2014). «"Je ne connais pas Ben Sadok". La genèse écrite du témoignage de Sartre au procès Ben Sadok ». Genesis, 39 : 57-69. 
BurguiÈre, A. (2006). L'École des Annales. Une histoire intellectuelle. Paris : Odile Jacob.

COHEN-SOlal, A. (1999). Sartre. 1905-1980 [1985]. Paris : Gallimard.

CORMAnN, G. (2012). «Émotion et réalité chez Sartre. Remarques à propos d'une anthropologie philosophique originale ». Bulletin d'Analyse Phénoménologique, 8 (1) : 286-302.

DOMENACH, J.-M. (1965). «L'enfance handicapée ». Esprit, 33 (343) : 577 580.

DOMENACH J.-M. et P. MARIE (1962). «Les enfants déformés et le "respect de la vie"». Esprit, 312 (12) : 1045-1063.

DUPONT, G. (2018). «Elles ont attendu 58 ans : les victimes Softenon gagnent en justice ». La Dernière Heure - Les Sports, $1^{\mathrm{er}}$ mars.

FANON, F. (2015). Écrits sur l'aliénation et la liberté. Éd. J. Khalfa et R. Young. Paris : La Découverte, 130-446.

— (2011). Les Damnés de la terre [1961]. In : Euvres. Paris : La Découverte, 660-672.

- (1959). L'An 5 de la révolution algérienne. Paris : Maspero.

— (1952). «Le "syndrome nord-africain" ». Esprit, 20 (187), 237-251.

- (1951). «La plainte du noir : l'expérience vécue du Noir ». Esprit, 19 (179), 657-679.

FEBVRE, L. (2009a). «Une vue d'ensemble. Histoire et psychologie » [1938]. In : Vivre l'histoire. Paris : Robert Laffont/Armand Colin, 180-191.

— (2009b). «La première renaissance française ; quatre prises de vue » [1925]. In : Vivre l'histoire, Paris : Robert Laffont/Armand Colin, 783-784.

- $(2009 \mathrm{c})$. «Comment reconstituer la vie affective d'autrefois ? La sensibilité et l'histoire» [1941]. In : Vivre l'histoire. Paris : Robert Laffont/Armand Colin, 146-157.

— (2009d). «Techniques, sciences et marxisme» [1935)]. In : Vivre l'histoire. Paris : Robert Laffont/Armand Colin, 853-854.

- (2009e). «La vie, cette enquête continue » [1935]. In : Vivre l'histoire. Paris : Robert Laffont/Armand Colin.

- (1995a). Combats pour l'histoire [1952]. Paris : Armand Colin.

- (1995b). «Une vue d'ensemble. Histoire et psychologie ». In : Combats pour l'histoire, [1952]. Paris : Armand Colin, 207-220.

- (1968). Le problème de l'incroyance au XVI siècle. La religion de Rabelais [1942 ; 1947]. Paris : Albin Michel.

— (1938). «Psychologie et histoire ». In : L'Encyclopédie française, t. VIII. La vie mentale, 8.12-3/8.12-7. 
FLANDRIN, J.-L. (1964). «Enfance et société [Ph. Ariès, L'enfant et la vie familiale sous l'ancien régime]». Annales. Économies, sociétés, civilisations, 19 (2), 322-329.

GAY, P. (1963). «Annales of Childhood ». Saturday Review, 23 mars, p. 7374.

GROS, G. (2013). « Philippe Ariès sous le regard de Joseph Czapski et de Pierre Vidal-Naquet pendant la guerre d'Algérie ». Histoire@Politique, 20 (2), p. 134-154.

- (2006). «Philippe Ariès, entre traditionalisme et mentalités. Itinéraire d'un précurseur ». Vingtième Siècle. Revue d'histoire, 90 (2), 121-140.

IUSO, A. (2010) «Enfances hors du temps ». L'Homme, 195-196, 103-123. Retrieved from : <https://journals.openedition.org/lhomme/22465>

MARIE, P. (1962). «Les bébés phoques ». Esprit, 30 (310), 497-500.

MAUSS, M. (1950). «Les techniques du corps » [1934, 1936]. In : Sociologie et Anthropologie. Paris : Presses Universitaires de France.

MERLEAU-PONTY, M. (2016). Entretiens avec Georges Charbonnier et autres dialogues. Lagrasse : Verdier, 333-391.

PAULUS J. et J. ROZET (1963). Le procès de la thalidomide. Paris : Gallimard.

PÉJU M. et P. PÉJU (2011). Le 17 octobre des Algériens suivi de G. Manceron, La triple occultation d'un massacre. Paris : La Découverte.

PonTALIS, J.-B. et F. DOLTO (1963). «Les mères ». Les Temps Modernes, 200 (février), 1272-1282.

ROLAND, E. (2017). Généalogie des dispositifs éducatifs en Belgique du XIVe au XXe siècle. Disciplinarisation et biopolitique de l'enfance : des grands schémas de la pédagogie à la science de l'éducation, Université Libre de Bruxelles (Thèse).

RomAn, J. (2002). Esprit. Écrire contre la guerre d'Algérie (1947-1962). Paris : Hachette.

SARTRE, J.-P. (2015). Les racines de l'éthique [1964]. Éd. J. Bourgault et G. Cormann. Études sartriennes, 19, 11-118.

- (2013). Qu'est-ce que la subjectivité ? Éd. M. Kail. Paris : Les prairies ordinaires.

- (2010a). «Merleau-Ponty» [1961]. In : Les Mots et autres écrits autobiographiques. Bibliothèque de la Pléiade. Paris : Gallimard.

- (2010b). "Cahier Lutèce». In: Les Mots et autres écrits autobiographiques. Bibliothèque de la Pléiade. Paris, Gallimard.

- (2005a). Questions de méthode [1957]. Paris : Gallimard.

- (2005b). « Morale et Histoire» [1965]. Éd. J. Simont et G. Cormann. Les Temps Modernes, 632-634, 268-414.

— (1987). «L'anthropologie » [1966]. In : Situations IX. Paris : Gallimard. 
— (1986). Questions de méthode. Éd. rev. et annotée par Arlette Elkaïm-Sartre. Paris : Gallimard.

- (1971-72). L'idiot de la famille : Gustave Flaubert de 1821 à 1857. Paris : Gallimard.

- (1939). Esquisse d'une théorie des émotions. Paris : Hermann.

TOUSSAINT, P. (1963). Le procès de Liège. Bruxelles : Éditions actuelles.

UlLOA, P.-M. (2012). « Les intellectuels et la guerre d'Algérie ». Esprit, mars.

Retrieved from: <https://esprit.presse.fr/actualites/marie-pierre-ulloa/lesintellectuels-et-la-guerre-d-algerie-39945\#_ftn14>

WAROWNY, V. (2002). Informer une thèse, ou l'information au service d'une thèse. Dynamique de l'argumentation médiatique dans l'affaire du Softénon. Mémoire de fin d'études en philologie romane, Université de Liège.

- (1999). Le Procès du Softenon : 5 novembre 1962 - 11 novembre 1962 : procès d'un assassinat ou procès d'un médicament? Mémoire de fin d'études en Sciences historiques, Université de Liège.

GRÉGORY CORMANN enseigne la philosophie sociale à l'Université de Liège. Il est spécialiste de la philosophie de Sartre, dont il a publié plusieurs inédits. Il est notamment l'éditeur, avec Jean Bourgault, de J.-P. Sartre, Les racines de l'éthique (Études sartriennes, $\mathrm{n}^{\circ}$ 19, Bruxelles, Ousia, 2015). Relisant la philosophie française contemporaine au double prisme de l'École française de sociologie et de la Théorie critique de l'École de Francfort, son travail a pour enjeu l'élaboration d'une anthropologie politique des émotions.

GRÉGORY CORMANN is associated professor of social philosophy at the University of Liège (Belgium). He is a specialist in Sartre's philosophy. He has published several Sartre's unpublished works. In particular, he edited, with Jean Bourgault: J.-P. Sartre, Les racines de l'éthique (Études sartriennes, $\mathrm{n}^{\circ} 19$, Bruxelles, Ousia, 2015). While reassessing French philosophy through the double prism of the French school of sociology and the critical theory outlined by the Frankfurt School, his work aims at elaborating a political anthropology of emotions. 